\title{
Cardiorenal Syndrome in Type 2 Diabetes Mellitus - Rational Use of Sodium-glucose Cotransporter-2 Inhibitors
}

Sanjay Kalra, ${ }^{1}$ Hasan Aydin, ${ }^{2}$ Manisha Sahay, ${ }^{3}$ Sujoy Ghosh, ${ }^{4}$ Sundeep Ruder, ${ }^{5}$ Mangesh Tiwaskar, ${ }^{6}$ Gary Kilov, ${ }^{7}$ Kamal Kishor, ${ }^{8}$ Tiny Nair, ${ }^{9}$ Vikas Makkar, ${ }^{10}$ Ambika Gopalakrishnan Unnikrishnan, ${ }^{11}$ Dinesh Dhanda, ${ }^{8}$ Nikhil Gupta, ${ }^{12}$ Bharath Srinivasan ${ }^{13}$ and Amit Kumar ${ }^{13}$

1. Bharti Hospital and Bharti Research Institute of Diabetes and Endocrinology (BRIDE), Karnal, India; 2. Department of Endocrinology and Metabolism, Yeditepe University School of Medicine, Istanbul, Turkey; 3. Department of Nephrology, Osmania Medical College and General Hospital, Hyderabad, Telangana, India; 4. Amri Hospital, Kolkata, India; 5. Life Fourways Hospital, University of the Witwatersrand, Cape Town, South Africa; 6. Shilpa Medical Research Center, Dahisar East, Mumbai, Maharashtra, India; 7. Department of General Practice, University of Melbourne, Melbourne, Australia; 8. Rama Superspeciality Hospital Karnal, Haryana, India; 9. Department of Cardiology, PRS Hospital, Trivandrum, Kerala, India; 10. Dayanand Medical College and Hospital, Ludhiana, India; 11. Chellaram Diabetes Hospital, Pune, India; 12. CanMed Multispeciality and Weight Management Clinics, Toronto, Canada; 13. Medical Affairs, AstraZeneca Pharma India Ltd, Bengaluru, Karnataka, India

DOI: https://doi.org/10.17925/EE.2020.16.2.113

\begin{abstract}
C ardiorenal syndrome (CRS) in people with type 2 diabetes mellitus (T2DM) illustrates the bidirectional link between the heart and the kidneys, with acute or chronic dysfunction of one organ adversely impacting the function of the other. Of the five subtypes identified, type 1 and 2 CRS occur because of the adverse impact of cardiac conditions on the kidneys. Type 3 and 4 occur when renal conditions affect the heart, and in type 5, systemic conditions impact the heart and kidneys concurrently. The cardiovascular and renoprotective benefits evidenced with sodium-glucose cotransporter-2 (SGLT2) inhibitors make them a potential choice in the management of CRS. Cardiovascular protection is mediated by a reduction in cardiac workload, blood pressure, and body weight; with improvement in lipid profile, uric acid levels, and adaptive ketogenesis process. Renoprotection is facilitated by reduction in albuminuria and hypoxic stress, and restoration of tubuloglomerular feedback. The favourable effect on cardiovascular complications and death, as well as renal complications and progression to end-stage kidney disease, has been confirmed in clinical trials. Guidelines endorse first-line use of SGLT2 inhibitors after metformin in patients with T2DM with high cardiovascular risk, chronic kidney disease or both. Since most trials with SGLT2 inhibitors excluded subjects with acute illness, patients with CRS subtypes 1 and 3 have not been studied adequately, making SGLT2 initiation in clinical practice challenging. Ongoing trials may provide evidence for SGLT2 inhibitor use in CRS. This review aims to enhance understanding of CRS and provide guidance for judicious use of SGLT2 inhibitors in T2DM.
\end{abstract}

\section{Keywords}

Diabetes mellitus, cardiorenal syndrome, sodium-glucose cotransporter-2 (SGLT2) inhibitors, cardiorenal protection, cardiovascular outcome trials

Disclosures: Bharath Srinivasan and Amit Kumar are employees of AstraZeneca Pharma India Ltd. Sanjay Kalra, Hasan Aydin, Manisha Sahay, Sujoy Ghosh, Sundeep Ruder, Mangesh Tiwaskar, Gary Kilov, Kamal Kishor, Tiny Nair, Vikas Makkar, Ambika Gopalakrishnan Unnikrishnan, Dinesh Dhanda and Nikhil Gupta have no financia or non-financial relationships or activities to declare in relation to this article. Sanjay Kalra is a member of the journal's Editorial Board.

Review Process: Double-blind peer review.

Acknowledgements: The authors would like to thank Dr Anita Bhat, MBBS, DTCD, of Covance Scientific Services \& Solutions Pvt Ltd, India, for providing medical writing assistance in the development of this manuscript, that was funded by AstraZeneca Pharma India Ltd in accordance with GPP3 guidelines.

Compliance with Ethics: This article involves a review of the literature and did not involve any studies with human or animal subjects performed by any of the authors.

Authorship: The named authors meet the International Committee

of Medical Journal Editors (ICMJE) criteria for authorship of this

manuscript, take responsibility for the integrity of the work as a whole,

and have given final approval for the version to be published.

Access: This article is freely accessible at touchENDOCRINOLOGY.com

(C) Touch Medical Media 2020.

Received: 25 February 2020

Accepted: 1 July 2020

Published Online: 6 October 2020

Citation: European Endocrinology. 2020;16(2):113-21

Corresponding Author: Sanjay Kalra, Bharti Research Institute of Diabetes and Endocrinology, Karnal, Haryana 132001, India. E: bridekn|@gmail.com

Support: No funding was received in the publication of this article.
The International Diabetes Federation (IDF) estimates that 463 million people worldwide suffer from diabetes, $90 \%$ of whom have type 2 diabetes mellitus (T2DM), and expects the total healthcare expenditure on diabetes to reach US $\$ 845$ billion by 2045 from US $\$ 760$ billion in 2019.1 People with diabetes comprise $9.3 \%$ of the world's adult population, and the IDF predicts that this number will rise to $10.9 \%$ ( 700 million) by $2045 .{ }^{1}$ People with T2DM have high morbidity and mortality, mainly because of the development of microvascular and macrovascular complications. Chronic kidney disease (CKD) is a major microvascular complication in T2DM. Data from the UK and the USA show that about 20\% and $40 \%$, respectively, of patients with diabetes develop CKD., ${ }^{2,3}$ Cardiovascular disease (CVD) is a multifactorial syndrome and is a leading cause of morbidity and mortality in T2DM. It has an overall prevalence of 32.2\%, and is responsible for $50.3 \%$ deaths globally. ${ }^{4}$ Its risk in a person with diabetes is enhanced by the presence of CKD.

Cardiorenal health is, therefore, the focus of attention in diabetes care. This has led to an important change in the American Diabetes Association (ADA) guidelines to include the 10-year atherosclerotic CVD risk as part of the overall assessment. ${ }^{5}$ The ADA 2019 new standards, with updates aimed at reducing myocardial infarction, strokes, heart failure and other manifestations of CVD in people with diabetes, have been endorsed by the American College of Cardiology (ACC). ${ }^{5}$ Other major guidelines like the ADA's - the European Association for the Study of Diabetes (EASD) consensus and the European Society of Cardiology (ESC) 2013 guidelines on diabetes, prediabetes, and CVDs developed in collaboration with 
EASD, also highlight the importance of assessment of cardiovascular risk, in addition to the prevention and management of CVD. ${ }^{6,7}$

The concept of cardiorenal syndrome (CRS) highlights the multiple links between the health of the kidneys and the heart. ${ }^{8}$ CRS is a complex disease affecting the heart and the kidneys simultaneously, with their harmful outcomes linked in a vicious cycle. The five subtypes of CRS identified are: acute CRS (type 1), chronic CRS (type 2), acute renocardiac syndrome (RCS) (type 3), chronic RCS (type 4), and secondary CRS (type 5). The term CRS indicates the bidirectional nature of the five subtypes. Patients with this dual burden are at an increased risk of hospitalisation, poor quality of life and increased mortality, thereby increasing the economic burden. ${ }^{8}$

Since CVD and CKD are considered as a homogenous entity by many clinicians, there is scope for misjudgement when they prescribe sodium-glucose cotransporter-2 (SGLT2) inhibitors for renal protection. The definition and classification of CRS provide a structure that helps clinicians understand the indications and contraindications for SGLT2 inhibitor use. T2DM is a key risk factor for the development of CRS; a strong association with type 2 CRS was reported in the National Health and Nutrition Examination Survey in the USA. ${ }^{9}$ Being a systemic condition with vascular complications impacting the heart and the kidneys, diabetes is also associated with type 4 and 5 CRS. ${ }^{10}$ Current regulations have helped explore the impact of antihyperglycaemic therapy on cardiovascular and renal health. The US Food and Drug Administration (FDA), from 2008 onwards, mandates that all new glucose-lowering agents run a cardiovascular outcome trial (CVOT) to grant approvals. ${ }^{11}$ This has helped us to understand the vascular protective potential of newer glucose-lowering agents, namely SGLT2 inhibitors and glucagon-like peptide-1 (GLP-1) agonists. ${ }^{12}$ Thus, the emergence of SGLT2 inhibitors and GLP-1 agonists offers the potential to provide cardiorenal protection over and above glycaemic control. Recent SGLT2-inhibitor CVOTs have provided more clarity, with the DECLARE-TIMI 58 trial (Dapagliflozin Effect on Cardiovascular Events-Thrombolysis in Myocardial Infarction 58 [ClinicalTrials. gov identifier: NCT01730534]) revealing the possibility of primary prevention of cardiorenal complications in T2DM. ${ }^{13}$ Similarly, the recent DAPA-HF study (Dapagliflozin And Prevention of Adverse-outcomes in Heart Failure; NCT03036124) results provide evidence for the likelihood of a new approach to manage subjects with heart failure and reduced ejection fraction (HFrEF), with and without T2DM. ${ }^{14}$ This narrative review utilises the concept of CRS to promote the rational use of SGLT2 inhibitors in T2DM, based on a literature search using PubMed and citation lists of relevant publications. Subject headings and keywords used included 'cardiorenal syndrome,' 'SGLT2i and cardiorenal syndrome,' and 'cardiorenal syndrome in type 2 diabetes mellitus.'

\section{Mechanism of action of SGLT2 inhibitors Mechanism of glycaemic control}

Four SGLT2 inhibitors - canagliflozin, dapagliflozin, empagliflozin, and ertugliflozin - have been approved by regulatory agencies in the USA, European Union and other parts of the world. Remogliflozin has been recently approved, but only in India. There are also three SGLT2 inhibitors that have approval only in Japan: ipragliflozin, luseogliflozin and tofogliflozin. Several other SGLT2 inhibitors are still under development. Figure 1 illustrates the SGLT2 inhibitor mechanism of action for glycaemic control and extraglyacemic metabolic effects.

SGLT2 inhibitors inhibit the reabsorption of glucose through the SGLT2 receptors expressed in the luminal surface of the proximal convoluted tubule (PCT) of the kidneys. The glucose threshold for urinary excretion increases in subjects with diabetes because of increased SGLT2 activity, and this threshold is lowered by the action of SGLT2 inhibitors. ${ }^{15}$ The resultant excretion of glucose in the urine causes plasma glucose levels to fall and results in glycaemic control. This mechanism is independent of insulin action and dependent on the blood glucose levels ensuring minimal potential for hypoglycaemia and beta-cell fatigue. ${ }^{16}$ The inhibition of sodium reabsorption (as it is cotransported with glucose), causes a small natriuresis and osmotic diuresis. ${ }^{17}$ Long-term glycaemic control by SGLT2 inhibitors is durable when used as monotherapy or in combination with other oral hypoglycaemic drugs. ${ }^{18,19}$

The vasculo-metabolic benefits of SGLT2 inhibitors include a reduction in blood pressure and body weight, along with an improvement in lipid profile and insulin sensitivity. The reduction in body weight ranges from 1 to $5 \mathrm{~kg}$, with a higher reduction in patients with long-standing diabetes and a higher baseline weight. ${ }^{20}$ The decreased weight is proven to be majorly (two-thirds) due to loss of fat mass, especially visceral fat, with a reduction in waist circumference. .1,22 $^{2}$ The reduction in blood pressure is seen with both the systolic and diastolic blood pressure components and is not associated with reactive tachycardia, hypotension, or syncope. ${ }^{23}$ Also, these effects are independent of the glycaemic and weight reduction benefits. The mechanism may initially be due to osmotic diuresis, but continuing benefits are probably because of local renin-angiotensin system inhibition. ${ }^{24}$ This was supported by an animal model study that demonstrated significant suppression of urine angiotensin II and angiotensinogen levels following treatment with dapagliflozin. ${ }^{25} \mathrm{~A}$ recent review reported an average blood pressure reduction of $4 / 2 \mathrm{mmHg}$, with a consistency between office and 24-hour ambulatory measurements, and a nocturnal systolic blood pressure reduction of $2.6 \mathrm{mmHg}$. These blood pressure reductions were even seen in patients already on treatment with angiotensin-converting-enzyme inhibitors or angiotensin ॥ receptor blockers. ${ }^{26}$ The effects on lipid profile are variable and include an increase in high-density-lipoprotein cholesterol (HDL-C), low-density-lipoprotein cholesterol (LDL-C) and a reduction in triglycerides. The ratio of $L D L-C$ to $H D L-C$, however, was not found to worsen. ${ }^{27,28}$ The chronic glycosuria by SGLT2 inhibitors with loss of calories, adipose tissue and weight improves insulin sensitivity.22,29

\section{Mechanism of cardiac protection}

In addition to improvement in cardiovascular risk factors like body weight, blood pressure, insulin resistance and lipid profile, calorie restriction mimicry and pro-ketogenic effect are the two mechanisms proposed in the Robin Hood hypothesis to explain the cardiovascular benefits. ${ }^{30}$ Robin Hood is remembered in English history for his act of stealing from the rich to support the poor. This is comparable to SGLT2 inhibitors, which give rest to the glucose metabolism, allowing the efficient utilisation of the unused store of body lipids for providing energy. The calorie restriction mimicry effect is mediated by the adenosine monophosphate-activated protein kinase (AMPK) pathway. ${ }^{30}$ The loss of calories in the urine by SGLT2 inhibitor action causes a shift in substrate utilisation from glucose to fat. This, in addition to being a mechanism for weight loss, is also a stimulant of AMPK activity with increased fatty acid oxidation in the skeletal muscles. This brings about an improvement in peripheral insulin sensitivity, with a reduction in endogenous, as well as exogenous, insulin requirement. ${ }^{31}$

Adaptive ketogenesis is another benefit attributed to a shift in metabolism from glucose to fat. SGLT2 inhibitors have a ketogenic action because of an increase in glucagon levels. ${ }^{30,32}$ The release of free fatty acids and their conversion in the liver to ketone bodies creates a favourable environment for myocardial metabolism. This explanation 
Figure 1: Mechanism of glycaemic and extraglycaemic actions of SGLT2 inhibitors

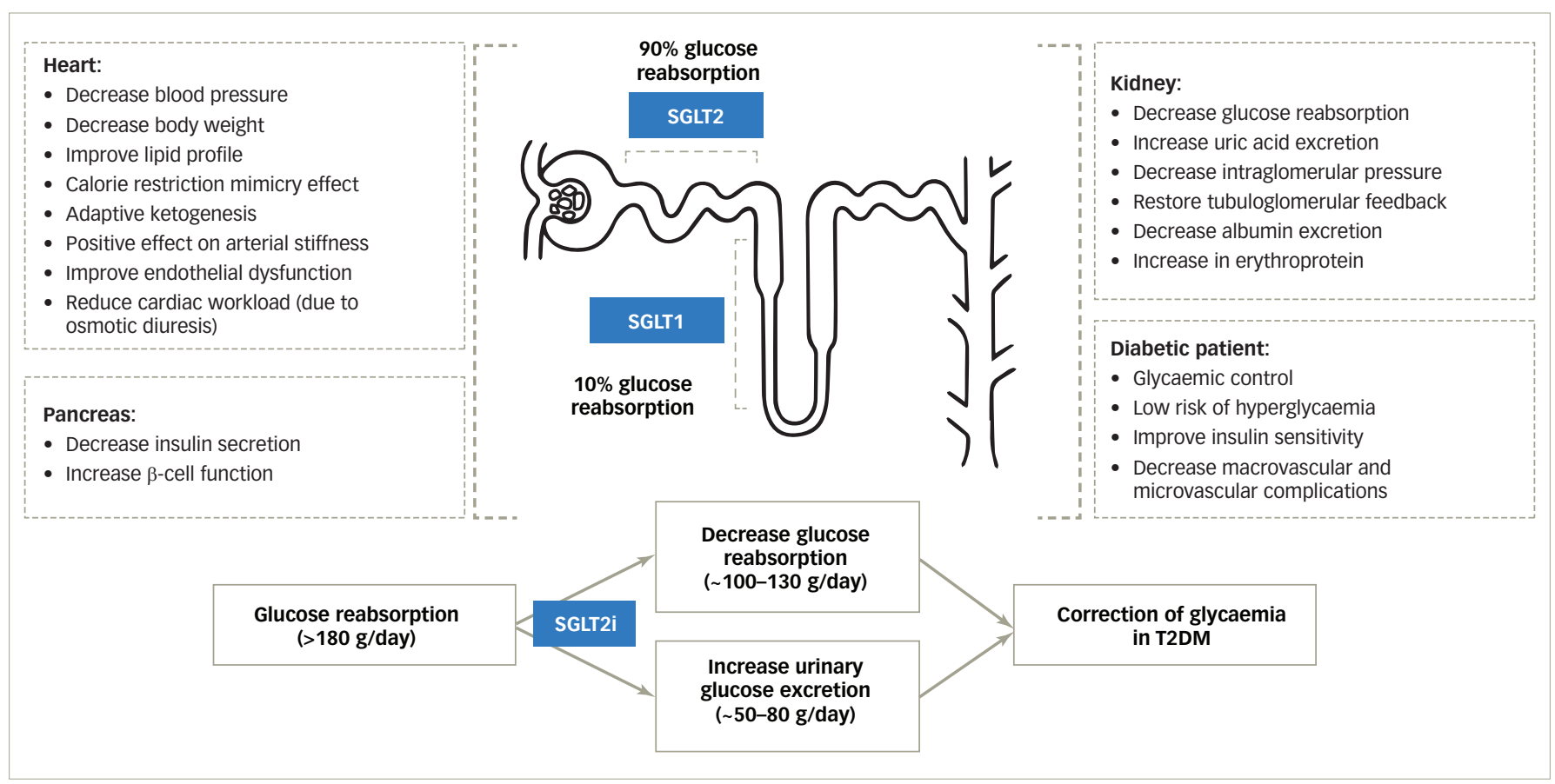

SGLT1 = sodium-glucose cotransporter-1; SGLT2 = sodium-glucose cotransporter-2; SGLTi = sodium-glucose cotransporter inhibitor; T2DM = type 2 diabetes mellitus.

for the cardiovascular benefit has been termed the 'thrifty substrate' hypothesis..$^{33}$ Ketones are akin to super food for the cardiac cells and considered superior to fatty acids and glucose for ensuring energy to the ischaemic diabetic myocardium. In addition, ketones reduce free radical-induced injury by decreasing the utilisation of free-fatty acids.

SGLT2 inhibitors are also said to have a positive effect on arterial stiffness and endothelial dysfunction by reducing oxidative stress independent of the blood pressure effect. This may also contribute to cardiovascular risk reduction. ${ }^{34}$ It is also hypothesised that a reduction in sodium and water retention, because of osmotic diuresis, leads to reduced ventricular filling pressures with resultant decrease in the cardiac workload, and therefore, better cardiac function. ${ }^{35}$

\section{Mechanism of renal protection}

Increased estimated glomerular filtration rate (eGFR) and microalbuminuria are markers of renal injury mediated by diabetes and hypertension. ${ }^{36,37}$ SGLT2 inhibitors decrease intraglomerular pressure by decreasing sodium reabsorption in the PCT and delivering increased sodium to the macula densa in the distal juxtaglomerular apparatus. This plays a vital role in restoring the tubuloglomerular feedback, reducing albuminuria and slowing the decline in renal function. ${ }^{38-40}$ The shift in metabolism to ketone bodies away from glucose and fat by SGLT2 inhibitors is hypothesised to lower renal oxygen consumption, alleviate hypoxic stress, improve renal function and slow the progression of diabetic kidney disease. Thus, the ketone super-fuel hypothesis has been found applicable to renal protection in addition to cardiac protection. ${ }^{41}$

The elevated uric acid levels in patients with diabetes can play a role in causing renal damage and other microvascular complications. ${ }^{42}$ SGLT2 inhibitors not only reduce plasma uric acid levels but also inhibit the renal reabsorption of sodium coupled-uric acid in the PCT, causing urinary excretion of uric acid. This lowering of uric acid helps in slowing the progression of CKD in addition to reducing adverse cardiovascular events. ${ }^{40}$
An increase in erythropoietin is indirectly attributed to SGLT2 inhibitors. Patients with diabetes are said to have compromised oxygenation in the PCT because of excessive glucose reabsorption. A reduction in nephrons' workload by SGLT2 inhibitors can aid the recovery of erythropoietin cells with haematocrit improvement and improved oxygen delivery, especially to the heart and kidneys. ${ }^{43}$

Reduction in oxidative stress with canagliflozin has been demonstrated to protect the kidneys from myocardial infarction-induced acute CRS (type 1) in type 2 diabetic rats. ${ }^{44}$ Through the above described mechanisms, SGLT2 inhibitors have been associated with slower progression to end-stage renal disease and reduced progression in the degree of albuminuria and eGFR, in addition to lower incidence of renal adverse events in several randomised controlled trials. ${ }^{45-48}$

\section{Place of SGLT2 inhibitor therapy in patients with type 2 diabetes with cardiovascular and renal risk}

SGLT2 inhibitors have developed an enviable evidence base with robust benefits on cardiovascular and renal outcomes. The benefits on reducing hospitalisation for heart failure (HHF) and progression of renal disease are proven regardless of existing atherosclerotic CVD or a history of heart failure. ${ }^{49}$

The cardiovascular and renal benefits of SGLT2 inhibitors are being evaluated in nine trials enrolling 64,817 patients. The EMPA-REG OUTCOME (EMPAgliflozin cardiovascular outcome event trial in type 2 diabetes mellitus patients-Removing Excess Glucose [NCT01131676]), CANVAS (CANagliflozin cardioVascular Assessment Study [NCT01032629]), CREDENCE (Canagliflozin and Renal Events in Diabetes with Established Nephropathy [NCT02065791]), and DECLARE-TIMI 58 are the four major completed trials in subjects with T2DM, with each of them reporting positive cardiovascular and renal outcomes (Table 1). The EMPA-Heart (Effects of Empagliflozin on Cardiac Structure in Patients With Type 2 Diabetes; NCT02998970) trial, 
Table 1: Results from landmark cardiovascular outcome trials with SGLT2 inhibitors

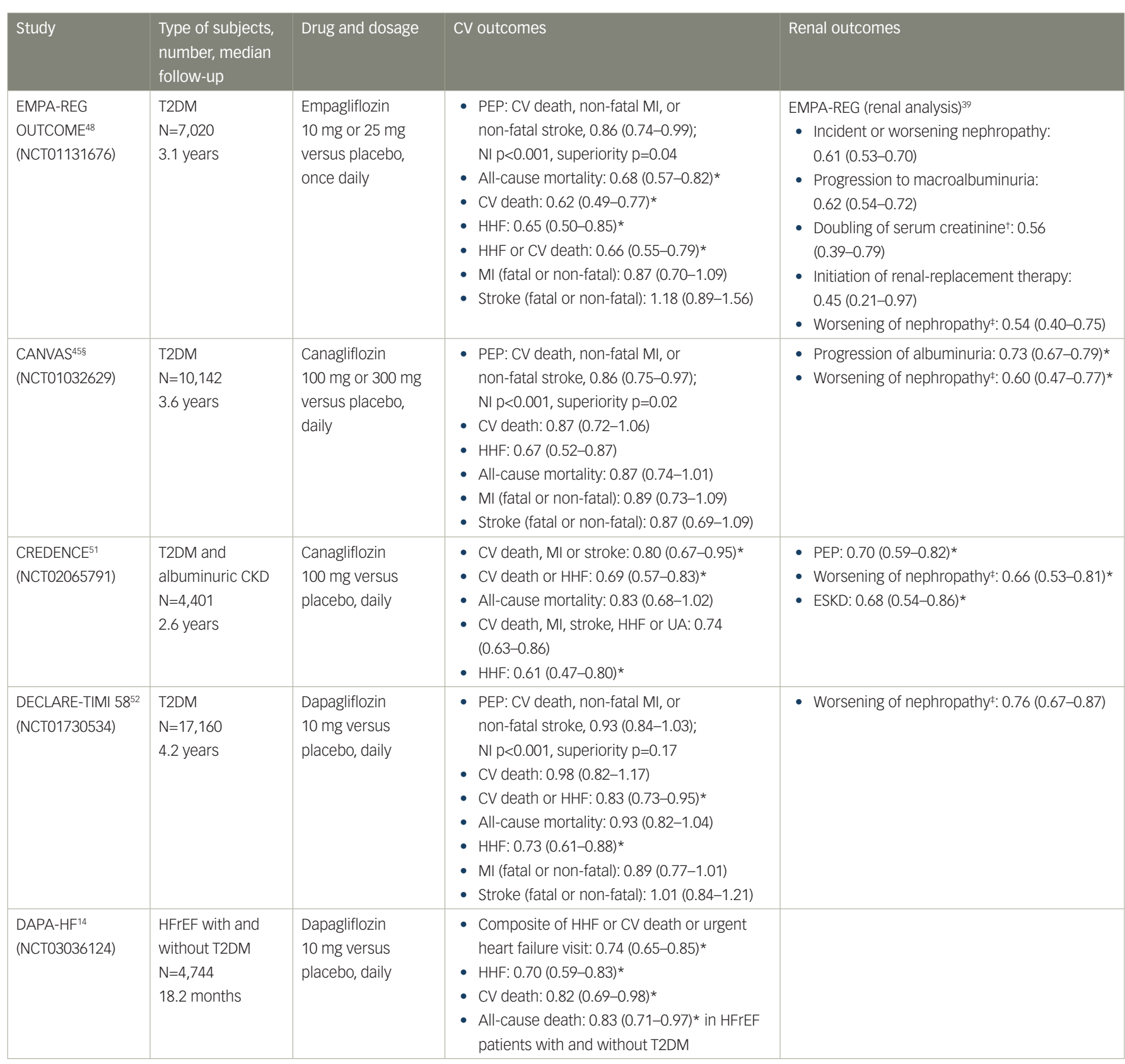

Outcomes reported as HR $(95 \% \mathrm{Cl})$.

* Significant.

${ }^{\dagger}$ Accompanied by eGFR of $\leq 45 \mathrm{~mL} / \mathrm{min} / 1.73 \mathrm{~m}^{2}$

Worsening nephropathy was defined as doubling of the serum creatinine level and an eGFR of $\leq 45 \mathrm{~mL} / \mathrm{min} / 1.73 \mathrm{~m}^{2}$, the need for continuous renal-replacement therapy, or death due to renal events in EMPA-REG OUTCOME; $40 \%$ reduction in eGFR, renal-replacement therapy, or death from renal causes in CANVAS; sustained decrease of $\geq 40 \%$ in eGFR to $<60 \mathrm{~mL} / \mathrm{min} / 1.73 \mathrm{~m}^{2}$, new end-stage renal disease, or death from any cause in DECLARE-TIMI 58; ESKD, doubling of the creatinine level, or renal death in CREDENCE.

spooled data from CANVAS and CANVAS-R.

CANVAS = CANagliflozin cardioVascular Assessment Study; CKD = chronic kidney disease; CREDENCE = Canagliflozin and Renal Events in Diabetes with Established Nephropathy Clinical Evaluation; CV = cardiovascular; DAPA-HF = Dapagliflozin And Prevention of Adverse-outcomes in Heart Failure; DECLARE-TIMI $58=$ Dapagliflozin Effect on Cardiovascular Events - Thrombolysis in Myocardial Infarction 58; eGFR = estimated glomerular filtration rate; EMPA-REG = EMPAgliflozin cardiovascular outcome event trial in type 2 diabetes mellitus patients - Removing Excess Glucose; ESKD = end-stage kidney disease; HFrEF = heart failure with reduced ejection fraction; HHF = hospitalisation for heart failure; HR = hazard ratio; $\mathrm{MI}=$ myocardial infarction; $\mathrm{NI}=$ non-inferiority; $P E P=$ primary end point; $S G L T 2=$ sodium-glucose cotransporter-2; T2DM = type 2 diabetes mellitus; UA= unstable angina

a follow-up of the main EMPA-REG OUTCOME trial, demonstrated a significant reduction $\left(-2.6\right.$ versus $-0.01 \mathrm{~g} / \mathrm{m}^{2}$ in placebo; $\left.\mathrm{p}=0.01\right)$ in left ventricular mass index, which is considered an independent predictor of cardiovascular events. ${ }^{50}$ The results of DAPA-HF, a study investigating benefits of dapagliflozin in HFrEF with T2DM (42\%) and without T2DM (58\%), were presented at the ESC Congress 2019. Over a median follow-up of 18.2 months, the composite of worsening heart failure or cardiovascular death was reduced significantly (hazard ratio [HR] 0.74; 95\% confidence interval [Cl] 0.65-0.85; $\mathrm{p}<0.00001$ ). In addition,
HHF (HR 0.70; 95\% Cl 0.59-0.83; $\mathrm{p}=0.00004$ ), cardiovascular death (HR 0.82; 95\% Cl 0.69-0.98; $p=0.029)$, and all-cause death (HR 0.83; $95 \% \mathrm{Cl} 0.71-0.97 ; p=0.022)$ reduced significantly. Quality of life, as measured by the Kansas City Cardiomyopathy Questionnaire score, was also significantly enhanced. The encouraging results from this study provide evidence for substantial cardiovascular risk reduction in subjects with HFrEF, irrespective of their diabetic status, by an SGLT2 inhibitor. ${ }^{14}$ The other four trials are ongoing and scheduled to be completed within the next year (Figure 2). 
Figure 2: Timeline of cardiovascular outcome trials with SGLT2 inhibitors

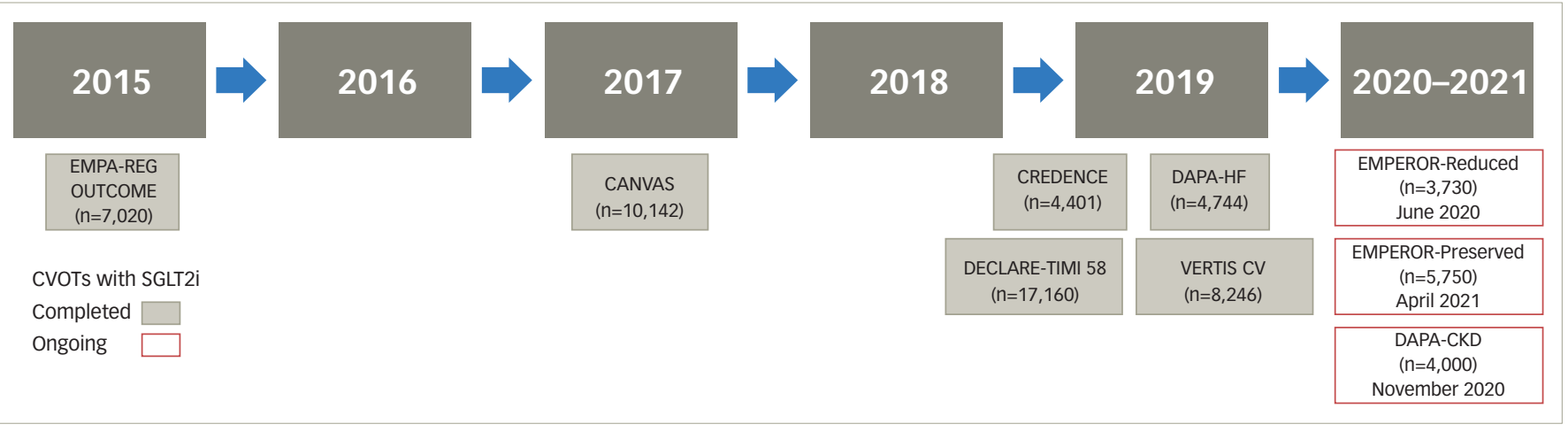

CANVAS = CANagliflozin cardiovascular Assessment Study; CREDENCE = Canagliflozin and Renal Events in Diabetes with Established Nephropathy Clinical Evaluation; CVOTS = cardiovascular outcomes trials; DAPA-CKD = Effect of Dapagliflozin on Renal Outcomes and Cardiovascular Mortality in Patients with Chronic Kidney Disease DAPA-HF = Dapagliflozin and Prevention of Adverse Outcomes in Heart Failure; DECLARE-TIMI $58=$ Dapagliflozin Effect on Cardiovascular Events - Thrombolysis in Myocardial Infarction 58; EMPA-REG Outcome = EMPAgliflozin cardiovascular outcome event trial in type 2 diabetes mellitus patients - Removing Excess Glucose; EMPEROR-Preserved = EMPagliflozin outcomE tRial in patients with chronic heaRt failure with preserved ejection fraction; EMPEROR-Reduced = EMPagliflozin outcomE tRial in patients with chronic heaRt failure with reduced ejection fraction; SGLT2i = sodium-glucose cotransporter 2 inhibtor; VERTIS CV = eValuation of ERTugliflozin effIcacy and Safety Cardiovascular outcomes trials.

Patients receiving SGLT2 inhibitor therapy (versus placebo) in the EMPA-REG OUTCOME, CANVAS, CREDENCE and DECLARE-TIMI 58 studies experienced lesser doubling of serum creatinine, incident dialysis, renal transplantations and/or renal deaths. ${ }^{43,47,5,52}$ The benefits shown in the randomised controlled trials were further substantiated with real-world evidence supporting its use in actual clinical practice in two observational trials. After applying the main inclusion criteria and outcomes of DECLARE-TIMI 58, the cardiovascular safety and event rates for dapagliflozin were compared with other glucose-lowering drugs in a nationwide study in Sweden. ${ }^{53}$ In this DECLARE-like cohort with 28,408 users, dapagliflozin reduced the HHF or cardiovascular mortality versus other glucose-lowering drugs by $21 \%$ (HR 0.79; $95 \%$ $\mathrm{Cl}$ 0.69-0.92). In addition, HHF separately was lower at HR 0.79 (95\% $\mathrm{Cl} 0.67-0.93)$ and cardiovascular mortality was lower at HR 0.75 (95\% $\mathrm{Cl}$ 0.57-0.97). A larger population was studied in the CVD-REAL study (NCT02993614) with data from 309,056 patients from six countries (USA, Norway, Denmark, Sweden, Germany and the UK). ${ }^{46}$ Patients initiated on SGLT2 inhibitors (canagliflozin, dapagliflozin, empagliflozin) versus other glucose-lowering drugs were compared to determine benefits in real-world practice. SGLT2 inhibitor use was associated with lower rates of HHF (HR 0.61; 95\% Cl 0.51-0.73), death (HR 0.49; 95\% Cl 0.41-0.57) and HHF or death (HR 0.54; 95\% $\mathrm{Cl} 0.48-0.60$ ).

\section{Recommendations and approvals for SGLT2 inhibitors to reduce cardiovascular and renal risk in patients with type 2 diabetes}

The ADA 2019 guidelines specified SGLT2 inhibitors (with proven CVD benefit, if eGFR adequate) or GLP-1 receptor agonists as first-line treatment after metformin when atherosclerotic CVD predominates (Level A recommendation), and also as a treatment of choice if a person with diabetes had coexisting heart failure or CKD (SGLT2 inhibitors with evidence of reducing heart failure and/or CKD: Level $C$ recommendation). ${ }^{5}$ Even without established atherosclerotic CVD or CKD, if there was a compelling need to minimise hypoglycaemia or weight gain, or even promote weight loss, SGLT2 inhibitors were ascribed a primary role. However, cost was recognised as an adverse factor for choosing an SGLT2 inhibitor. The ADA-EASD 2020 consensus, published recently, updated this further by recommending SGLT2 inhibitor pharmacotherapy in high-risk individuals with established T2DM, to reduce major adverse cardiovascular events (MACE), HHF, cardiovascular death or CKD progression, independent of the baseline glycosylated haemoglobin (HDA1C) or personalised HbA1C goals. Furthermore, SGLT2 inhibitor recommendation was strengthened for people with T2DM and heart failure (especially those with HFrEF) to reduce MACE, $\mathrm{HHF}$ and cardiovascular death, and for people with T2DM and CKD (eGFR $30-60 \mathrm{~mL} / \mathrm{min} / 1.73 \mathrm{~m}^{2}$ or urinary albumin-to-creatinine ratio $>30 \mathrm{mg} / \mathrm{g}$, particularly $>300 \mathrm{mg} / \mathrm{g}$ ) to prevent progression of CKD, HHF, MACE and cardiovascular death. ${ }^{54}$

The AHA also recognises the cardiovascular benefits with SGLT2 inhibitors and recommends them for the reduction of cardiovascular risk in T2DM. ${ }^{55}$ The recommendation is to initiate an SGLT2 inhibitor in adults with T2DM and additional atherosclerotic CVD risk factors who require glucose-lowering therapy despite initial lifestyle modifications and metformin. A recent scientific statement from the AHA that focused on classification, pathophysiology, diagnosis and treatment strategies in CRS mentions SGLT2 inhibitors as one of the glucose-lowering therapies with positive cardiorenal outcomes in T2DM. ${ }^{56}$

At the same time, there is a concern about the glucose-lowering benefit and safety of SGLT2 inhibitors in people with renal impairment. This review uses the taxonomic format of CRS to share a pragmatic approach to SGLT2 inhibitor prescription.

\section{Understanding cardiorenal syndrome}

CRS defines the two-way pathological relationship between the heart and kidneys, whereby acute or chronic dysfunction in one organ may induce acute or chronic dysfunction of the other in the presence of vascular dysfunction. ${ }^{57}$ Five subtypes of the syndromes have been identified and can be encountered in diabetes. ${ }^{8}$ It is possible that patients may transition between the CRS subtypes during the course of the disease, making the classification and management of CRS complex. ${ }^{57}$

i. Acute CRS (type 1): this subtype involves acute worsening of heart function as in acute heart failure, acute coronary syndrome (ACS), right ventricular failure, or cardiogenic shock leading to acute kidney injury (AKI) and/or dysfunction. Mechanisms include renin-angiotensin-aldosterone system (RAAS) and sympathetic nervous system (SNS) activation with efferent arteriole vasoconstriction reducing the renal perfusion pressure and eGFR. About $27-40 \%$ of patients hospitalised for acute decompensated heart failure (ADHF) develop AKI and come under this category, and account for $50 \%$ of all CRS cases. ${ }^{58,59}$ 
ii. Chronic CRS (type 2): chronic heart failure can lead to a chronic state of kidney disease and has been reported in 63\% of patients hospitalised for chronic heart failure. ${ }^{60}$ Twenty percent of all CRS cases fall under this category. Chronic renal hypoperfusion and raised venous pressure are said to contribute to renal dysfunction with progression to CKD. ${ }^{57}$

iii. Acute RCS (type 3): this subtype refers to AKI and renal ischaemia leading to heart injury and/or dysfunction with a mechanism that includes volume overload, toxaemia, humoral mediators and electrolyte imbalance, along with RAAS and SNS activation. Examples include development of ACS, arrhythmia, or acute heart failure after the onset of AKI. ${ }^{57}$

iv. Chronic RCS (type 4): this subtype refers to CKD leading to heart injury, disease and/or dysfunction. Cardiac disease in CKD is commonly manifested as chronic heart failure or diastolic dysfunction and is associated with 10 to 20 -fold higher cardiac-specific mortality rates, as compared with non-CKD populations. ${ }^{57,61}$ Anaemia, electrolyte imbalance, uremic toxins, chronic inflammation and oxidative stress are considered some of the mechanisms in CKD stages 3-6, while T2DM and hypertension can predispose to cardiac dysfunction in CKD stages 1 and $2 .^{10}$

v. Secondary CRS (type 5): acute or chronic systemic conditions, like sepsis, diabetes, cirrhosis and amyloidosis causing simultaneous injury and/or dysfunction to the heart and kidneys, fall into this category. The mechanism includes a combination of RAAS and SNS activation, inflammatory mediators release, toxaemia, oxidative stress and haemodynamic dysfunction. ${ }^{10}$ Treatment of the primary systemic illness, in general, improves heart and kidney function.

\section{Pathophysiology of cardiorenal syndrome}

Multiple interrelated mechanisms contribute to the development of CRS and its progression. Activation of RAAS and SNS to compensate for a fall in cardiac output, and renal hypoperfusion leads to volume overload, cardiac hypertrophy and fibrosis, and impaired tubuloglomerular feedback with renal hypoxia and injury. A cycle of inflammation, oxidative stress and vascular endothelial dysfunction is set into play by this neurohormonal activation and results in complex pathophysiological cross-talks between the heart and kidneys, leading to a bidirectional organ dysfunction. ${ }^{57} \mathrm{~A}$ single 'CRS umbrella' concept, where acute and chronic cardiovascular events increase risk for AKI and CKD, and acute and chronic renal events increase risk of new cardiovascular events in T2DM, was ideated in a recent editorial. ${ }^{62}$

\section{General principles of cardiorenal syndrome management}

Pharmacologic management involves pragmatic use of RAAS inhibitors, beta-blockers, mineralocorticoid receptor antagonists, diuretics, inotropes and vasodilators in the absence of formal evidence-based guidelines for CRS management. Optimal management of the underlying systemic conditions, such as hypertension, diabetes, sepsis and anaemia, are also of prime importance. Non-pharmacologic management includes the use of devices (left ventricular assist device, haemodynamic sensors), dialysis to support renal function, and transplant (heart, kidney, or even dual organ transplant) for end-stage patients. Early and effective diagnosis with the use of biomarkers (interlukin-18, soluble suppression of tumourigenicity 2, cystatin C, neutrophil gelatinase-associated lipocalin, cardiac troponin, urinary $\mathrm{C}$-type natriuretic peptide, angiopoietin 2, soluble thrombomodulin, microRNA) and/or imaging (cardiac/renal ultrasound, magnetic resonance imaging, etc.) may be the key to achieving success in CRS management, while palliative care to address psychosocial issues is important to reduce suffering and improve quality
Figure 3: The role of SGLT2 inhibitors in type 2 diabetes mellitus with cardiorenal syndrome

Reduces renin-angiotensin-aldosterone system induced neurohormonal and sympathetic activation

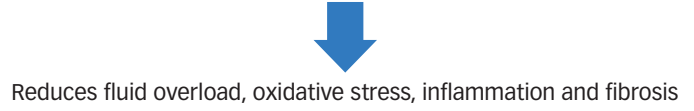
in heart and kidney

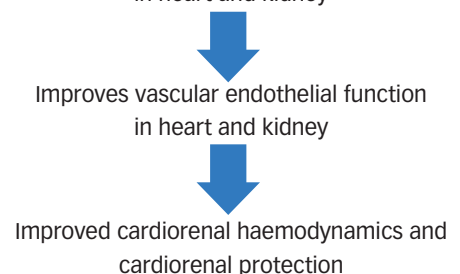

cardiorenal protection

of life in people with advanced CRS. ${ }^{57}$ SGLT2 inhibitors, by way of their pleiotropic mechanism of action with beneficial effects on HHF, MACE, CKD, cardiovascular and all-cause mortality in people with and without diabetes, offer a novel way of managing CRS (Figure 3) on top of existing therapies and are arousing clinical interest worldwide. It is therefore important to understand the patient selection criteria for the appropriate use of SGLT2 inhibitors in CRS.

\section{Patient selection criteria and rationale for prescribing SGLT2 inhibitors}

SGLT2 inhibitors are currently being prescribed in the following clinical situations

i. T2DM with CKD: renal analyses of the EMPA-REG, CANVAS, CREDENCE and DECLARE-TIMI 58 studies have demonstrated reno-protective benefits with SGLT2 inhibitors. ${ }^{33}$ Some ongoing/recently concluded SGLT2 inhibitor studies have lowered the eGFR threshold for SGLT2 initiation to 20,25, and $30 \mathrm{~mL} / \mathrm{min} / 1.73 \mathrm{~m}^{2}$ for empagliflozin (EMPA-Kidney [NCT03594110]), dapagliflozin (DAPA-CKD [NCT03036150]), and canagliflozin (CREDENCE), respectively. ${ }^{64-66}$

ii. T2DM with chronic heart failure: the EMPA-REG OUTCOME trial was the first to demonstrate significant cardioprotective benefits in this population. The significant 35\% reduction in HHF rationalised that SGLT2 inhibition could be an important strategy for treating patients with heart failure, with or without diabetes. The CANVAS program, real-life data from the CVD-REAL Study, and DECLARE-TIMI 58 added strength to this evidence, suggesting that the observed benefit is a class effect for SGLT2 inhibitors. ${ }^{41,42,46}$ It is equally important to understand the limitations and contraindications of SGLT2 inhibitors to avoid unwarranted use and unwanted adverse effects.

\section{SGLT2 inhibitors are currently not being prescribed in the following clinical situations}

i. T2DM with AKI: the chances of AKI with two of the three approved SGLT2 inhibitors (canagliflozin and dapagliflozin) were brought up in an FDA alert in December 2015. ${ }^{67}$ This FDA warning was strengthened in June 2016. However, a real-world study could not substantiate this increased risk of AKI with SGLT2 inhibitors (specifically canagliflozin and dapagliflozin) over a median follow-up of 14 months. ${ }^{68} \mathrm{~A}$ meta-analysis of 11 randomised controlled trials proved a protective effect of SGLT2 inhibitors for AKI, an effect primarily driven by the evidence with empagliflozin. ${ }^{69}$ AKI is a challenging condition to manage and adds to the burden by increasing the need for renal replacement therapy, hospital stays and costs, and leads to worse patient outcomes. Prerenal is the most common 
Table 2: Estimated glomerular filtration-based recommendations for SGLT2 inhibitors

\begin{tabular}{|c|c|c|c|}
\hline SGLT2i molecule & Patient type & eGFR threshold & Recommendation \\
\hline \multirow[t]{5}{*}{ Canagliflozin ${ }^{77 *}$} & \multirow[t]{3}{*}{ Renal impairment } & $60-<90 \mathrm{~mL} / \mathrm{min} / 1.73 \mathrm{~m}^{2}$ & $\begin{array}{l}\text { No dose adjustment is needed. Recommended starting dose } \\
100 \text { mg once daily }\end{array}$ \\
\hline & & $<60 \mathrm{~mL} / \mathrm{min} / 1.73 \mathrm{~m}^{2}$ & $\begin{array}{l}\text { Should not be initiated. If already on treatment, dose should be } \\
\text { reduced to } 100 \text { mg/day }\end{array}$ \\
\hline & & $<45 \mathrm{~mL} / \mathrm{min} / 1.73 \mathrm{~m}^{2}$ & Discontinue canagliflozin \\
\hline & ESRD or on dialysis & - & Canagliflozin should not be used \\
\hline & $\begin{array}{l}\text { CVD/history of hypotension on } \\
\text { antihypertensive/on diuretic/ } \geq 75 \text { years of age }\end{array}$ & $<60 \mathrm{~mL} / \mathrm{min} / 1.73 \mathrm{~m}^{2}$ & Caution is advised \\
\hline \multirow[t]{4}{*}{ Empagliflozin ${ }^{78 \dagger}$} & \multirow[t]{3}{*}{ Renal impairment } & $60-<90 \mathrm{~mL} / \mathrm{min} / 1.73 \mathrm{~m}^{2}$ & $\begin{array}{l}\text { No dose adjustment is needed. Recommended starting dose } \\
10 \text { mg once daily }\end{array}$ \\
\hline & & $<60 \mathrm{~mL} / \mathrm{min} / 1.73 \mathrm{~m}^{2}$ & $\begin{array}{l}\text { Should not be initiated. If already on treatment, dose should be } \\
\text { reduced to } 10 \mathrm{mg} / \text { day }\end{array}$ \\
\hline & & $<45 \mathrm{~mL} / \mathrm{min} / 1.73 \mathrm{~m}^{2}$ & Discontinue empagliflozin \\
\hline & ESRD or on dialysis & - & Empagliflozin should not be used \\
\hline \multirow[t]{2}{*}{ Dapagliflozin $79 \neq$} & \multirow[t]{2}{*}{ Renal impairment } & $>45-<60 \mathrm{~mL} / \mathrm{min} / 1.73 \mathrm{~m}^{2}$ & No dose adjustment \\
\hline & & $<45 \mathrm{~mL} / \mathrm{min} / 1.73 \mathrm{~m}^{2}$ & Discontinue dapagliflozin \\
\hline \multirow[t]{2}{*}{ Ertugliflozin ${ }^{80}$} & \multirow[t]{2}{*}{ Renal impairment } & $<60-30 \mathrm{~mL} / \mathrm{min} / 1.73 \mathrm{~m}^{2}$ & Use of ertugliflozin is not recommended \\
\hline & & $<30 \mathrm{~mL} / \mathrm{min} / 1.73 \mathrm{~m}^{2}$ & Ertugliflozin is contraindicated \\
\hline Remogliflozin ${ }^{81}$ & Renal impairment & - & $\begin{array}{l}\text { Adjustment of the dosage of remogliflozin in subjects with mild or } \\
\text { moderate renal impairment is not indicated }\end{array}$ \\
\hline
\end{tabular}

* Monitoring of renal function: prior to initiation of canagliflozin and at least annually, thereafter; prior to initiation of concomitant medicinal products that may reduce renal function and periodically thereafter; for renal function approaching moderate renal impairment, at least 2-4 times per year. If renal function falls persistently below eGFR $45 \mathrm{~mL} / \mathrm{min} / 1.73 \mathrm{~m}^{2}$ or $\mathrm{CrCl}<45 \mathrm{~mL} / \mathrm{min}$, canagliflozin treatment should be discontinued.

${ }^{\dagger}$ Monitoring of renal function: due to the mechanism of action, the glycaemic efficacy of empagliflozin is dependent on renal function. Therefore, assessment of renal function is recommended as follows: prior to empagliflozin initiation, and periodically during treatment (at least yearly); prior to initiation of any concomitant product that may have a negative impact on renal function.

${ }^{\ddagger}$ Monitoring of renal function: prior to initiation of dapagliflozin and at least yearly thereafter; prior to initiation of concomitant medicinal products that may reduce renal function and periodically thereafter; for renal function with eGFR $<60 \mathrm{~mL} / \mathrm{min}$, at least 2-4 times per year.

$\mathrm{CrCl}=$ creatinine clearance; $C V D=$ cardiovascular disease; eGFR = estimated glomerular filtration rate; ESRD = end stage renal disease;

SGLT2i = sodium-glucose cotransporter-2 inhibitor.

cause of AKI in patients hospitalised for cardiac interventions with an increased workload and oxygen consumption in the PCT. ${ }^{70}$ The consequent tubulointerstitial hypoxia is said to be mitigated by SGLT2 inhibitors. The improved renal cortical oxygen tension demonstrated in a study in diabetic rats, may play a role in maintaining tubular cell integrity and preventing proteinuria. ${ }^{71}$ There is a need for preclinical and clinical studies designed to focus on all aspects of AKI and not merely only on GFR biomarkers, to further understand these effects of SGLT2 inhibitors. ${ }^{72}$

ii. T2DM with ACS: though there is broad consensus (from the ADA 2019 guidelines) regarding the use of SGLT2 inhibitors in stable coronary artery disease, there is no clear guidance regarding their use during and immediately after ACS. ${ }^{5}$ No study so far has evaluated the use of SGLT2 inhibitors in the early post-ACS period. Presently, SGLT2 inhibitors should not be initiated during or upon discharge from ACS care. However, they should continue if they have already been prescribed, provided there are no contraindications to use. Use of SGLT2 inhibitors should be considered at the first post-ACS outpatient follow-up visit and monitored per routine practice. ${ }^{73}$

iii. During HHF: the safety and efficacy of SGLT2 inhibitors in ADHF is still under evaluation. In a single-centre retrospective study in Japan involving patients with T2DM hospitalised for ADHF, the use of SGLT2 inhibitors was superior to conventional therapy for prevention of $\mathrm{AKI}^{74}$ However, more studies will be required to confirm this result. Considering the unstable haemodynamics and variable renal function in ADHF, SGLT2 inhibitors are at present restricted to stable patients in the outpatient setting.
The recommendations and expert consensus to use SGLT2 inhibitors are based on the results of various randomised controlled trials. While these trials may differ in the study design, they all share one common factor: all studies recruited stable, chronic participants without any acute illness and significant nondiabetic comorbidity. ${ }^{75}$ This means that patients in the CRS categories 1, 2, 3, and 4 are excluded from most of the trials. CRS 1 and 3 classes are even more niche with sparse evidence, making initiation of SGLT2 inhibitors in acute situations challenging.

\section{Pragmatic rationale for SGLT2 inhibitor use in cardiorenal syndrome}

Since SGLT2 inhibitors have not been studied to show cardiovascular safety in those with impaired renal function, simplified guidance is needed for the safe use of SGLT2 inhibitors in CRS. ${ }^{76}$ Tables $2^{77-81}$ and $3^{63,64,81-86}$ describe pragmatic suggestions regarding initiation and continuation of SGLT2 inhibitors in different CRS subtypes and the various stages of AKI. SGLT2 inhibitors should not be initiated in the setting of CRS 1 and 3,but can be continued with close haemodynamic and renal-function monitoring. In CRS 2, 4, and 5, SGLT2 inhibitors are preferred drugs for glycaemic, as well as metabolic, control. They should be prescribed in specific situations, per the local approved prescribing information. ${ }^{77-87}$

Patients with an adequate eGFR may take SGLT2 inhibitors safely, while those with lower eGFR should be monitored closely. The eGFR cut-offs vary from drug to drug in the same class (Table 2), and from 
Table 3: SGLT2 inhibitor usage in various medical conditions

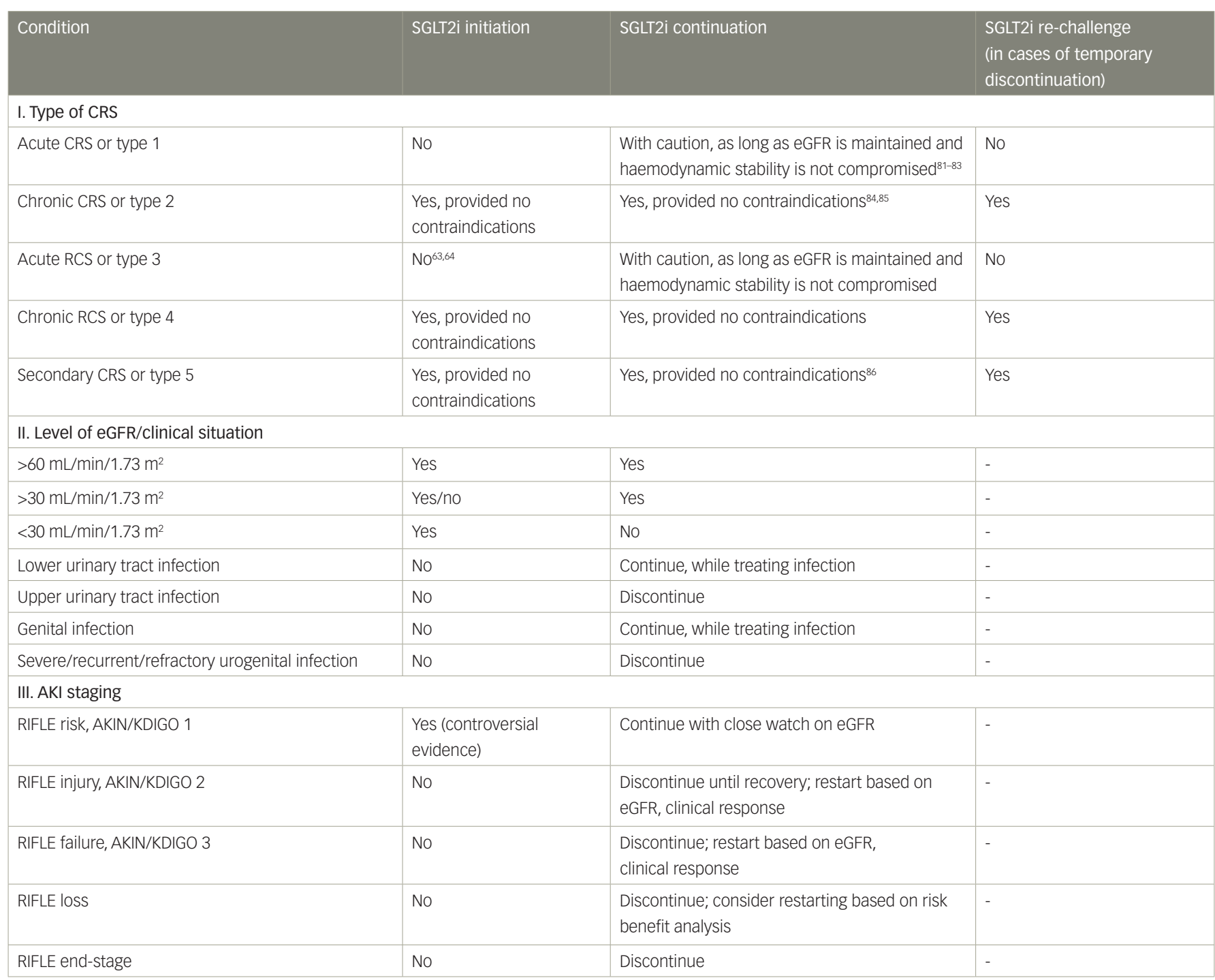

AKI = acute kidney injury; $A K I N=$ acute kidney injury network; $C R S$ = cardiorenal syndrome; eGFR = estimated glomerular filtration rate; $K D I G O=$ Kidney Disease Improving Global Outcomes; RCS = renocardiac syndrome; RIFLE = risk, injury and failure, and loss, and end-stage kidney disease; SGLT2i = sodium-glucose cotransporter-2 inhibitor.

country to country. Hence, prescribers should be aware of their national guidelines for prescribing decisions. SGLT2 inhibitors should not be initiated during an active bout of urinary or genital tract infection, and may be discontinued in patients with severe, refractory, or recurrent genitourinary infection. Care should be taken to avoid SGLT2 inhibitor use in acute medical and surgical conditions, which includes sepsis.

\section{Conclusion}

The management of diabetes has moved from the singular focus of glycaemic control to cover the wider patient-centric objectives of cardiorenal protection and reduction in morbidity and mortality. The glucose-dependent and insulin-independent glycaemic control by SGLT2 inhibitors ensures minimal hypoglycaemia and beta-cell protection. The pleiotropic benefits mediate weight loss, blood pressure reduction, uric acid reduction, favourable myocardial metabolism, and restoration of tubuloglomerular feedback. The collaborative effect of these pleiotropic benefits can help to achieve the broader objectives of cardiorenal protection.

CRS forms a crucial link between the heart and the kidneys and this article explored the regulated and rational use of SGLT2 inhibitors. The development of efficient treatment strategies requires a comprehensive understanding of the cardiorenal cross-talk in CRS. Although SGLT2 inhibitors form a rational addition to the treatment armamentarium for managing CRS, the results of the ongoing CVOT trials will provide more concrete evidence. However, optimum patient selection is vital for maximising the cardiorenal benefits provided by SGLT2 inhibitors, taking care to avoid their use in acute CRS. Thus, rational and responsible use of SGLT2 inhibitors, with a good understanding of the underlying CRS pathology, while differentiating acute from chronic illness, may contribute to a reduction in cardiorenal complications and improve the quality of life of people with T2DM. •
1. International Diabetes Federation. IDF Diabetes Atlas $9^{\text {th }}$ Edition, 2019. Available at: www.diabetesatlas.org (accessed 25 July 2020).

2. Dean J. Organising care for people with diabetes and renal disease. J Ren Care. 2012;38(Suppl. 1):23-9.

3. Bailey RA, Wang Y, Zhu V , et al. Chronic kidney disease in US adults with type 2 diabetes: an updated national estimate of prevalence based on Kidney Disease: Improving Global Outcomes (KDIGO) staging. BMC Res Notes. 2014;7:415. 4. Einarson TR, ACS A, Ludwig C, Panton UH. Prevalence of cardiovascular disease in type 2 diabetes: a systematic literature review of scientific evidence from across the world in
2007-2017. Cardiovasc Diabetol. 2018:17:83 Summary of revisions: standards of medical care in diabetes-2019. Diabetes Care. 2019;42(Suppl. 1):S4-6. Davies MJ, D'Alessio DA, Fradkin J, et al. Management of hyperglycaemia in type 2 diabetes, 2018. A consensus report by the American Diabetes Association (ADA) and the European 
Association for the Study of Diabetes (EASD). Diabetologia. 2018;61:2461-98

7. Rydén L, Grant PJ, Anker SD, et al. ESC Guidelines on diabetes, pre-diabetes, and cardiovascular diseases developed in collaboration with the EASD: the task force on diabetes, pre-diabetes, and cardiovascular diseases of the European Society of Cardiology (ESC) and developed in collaboration with the European Association for the Study of Diabetes (EASD). Eur Heart J. 2013;34:3035-87.

8. Ronco C, McCullough P, Anker SD, et al. Cardio-renal syndromes: report from the consensus conference of the acute dialysis quality initiative. Eur Heart J. 2010;31:703-11.

9. Banerjee S, Panas R. Diabetes and cardiorenal syndrome: understanding the "triple threat". Hellenic I Cardiol. 2017;58:342-7.

10. Raina R, Nair N, Chakraborty R, et al. An update on the pathophysiology and treatment of cardiorenal syndrome. Cardiol Res. 2020:11:76-88.

11. Federal Register. Guidance for industry on diabetes mellitus-evaluating cardiovascular risk in new antidiabetic therapies to treat type 2 diabetes. 2008 . Available at: www.federalregister.gov/documents/2008/12/19/E8-30086/ guidance-for-industry-on-diabetes-mellitus-evaluatingcardiovascular-risk-in-new-antidiabetic (accessed 25 July 2020).

12. Tong L, Adler S. Glycemic control of type 2 diabetes mellitus across stages of renal impairment: information for primary care providers. Postgrad Med. 2018;130:381-93.

13. Connelly KA, Bhatt DL, Verma S. Can we DECLARE a victory against cardio-renal disease in diabetes? Cell Metab. 2018;28:813-5.

14. McMurray JJV, Solomon SD, Inzucchi SE, et al. DAPA-HF trial committees and investigators. dapagliflozin in patients with heart failure and reduced ejection fraction. N Eng/ I Med. 2019:381:1995-2008.

15. Heerspink HJ, Perkins BA, Fitchett DH, et al. Sodium glucose cotransporter 2 inhibitors in the treatment of diabetes mellitus: cardiovascular and kidney effects, potential mechanisms, and clinical applications. Circulation. 2016:134:752-72.

16. Kalra S. Sodium-glucose co-transporter-2 (SGLT2) inhibitors: a review of their basic and clinical pharmacology. Diabetes Ther 2014;5:355-66.

17. Moses RG, Colagiuri S, Pollock C. SGLT2 inhibitors: new medicines for addressing unmet needs in type 2 diabetes. Australas Med J. 2014;7:405-15.

18. Leiter $L A$, Yoon $\mathrm{K}-\mathrm{H}$, Arios $\mathrm{P}$, et al. Canagliflozin provides durable glycemic improvements and body weight reduction over 104 weeks versus glimepiride in patients with type 2 diabetes on metformin: a randomized, double blind, phase 3 study. Diabetes Care. 2015;38:355-64.

19. Prato SD, Nauck M, Duran-Garcia S, et al. Long term glycaemic response and tolerability of dapagliflozin versus a sulfonylurea as add-on therapy to metformin in patients with type 2 diabetes: 4-year data. Diabetes Obes Metab. 2015:17:581-90.

20. Singh AK, Unnikrishnan AG, Zargar AH, et al. Evidence-based consensus on positioning of SGLT2i in type 2 diabetes mellitus in Indians. Diabetes Ther. 2019;10:393-428.

21. Rosenstock J, Jelaska $A$, Wang $F$, et al. Empagliflozin as add-on to basal insulin for 78 weeks improves glycemic control with weight loss in insulin-treated type 2 diabetes (T2DM). Can J Diabetes. 2013;37:S32.

22. Tosaki T, Kamiya H, Himeno T, et al. Sodium-glucose co-transporter 2 inhibitors reduce the abdominal visceral fat area and may influence the renal function in patients with type 2 diabetes. Intern Med. 2017;56:597-604

23. Vasilakou D, Karagiannis $T$, Athanasiadou $E$, et al. Sodium-glucose cotransporter 2 inhibitors for type 2 diabetes: a systematic review and meta-analysis. Ann Intern Med. a systematic review

24. Lambers HHJ, de Zeeuw D, Wie L, et al. Dapagliflozin a glucose-regulating drug with diuretic properties in subjects with type 2 diabetes. Diabetes Obes Metab. 2013;15:853-62.

25. Shin SJ, Chung S, Kim SJ, et al. Effect of sodium-glucose co-transporter 2 inhibitor, dapagliflozin, on renal renin-angiotensin system in an animal model of type 2 diabetes. PLOS One. 2016;11:e0165703.

26. Sternlicht $\mathrm{H}$, Bakris GL. Blood pressure lowering and sodium-glucose co-transporter 2 inhibitors (SGLT2is): more than osmotic diuresis. Curr Hypertens Rep. 2019;21:12.

27. Inzucchi SE, Zinman B, Wanner C, et al. SGLT-2 inhibitors and cardiovascular risk: proposed pathways and review of ongoing outcome trials. Diab Vasc Dis Res. 2015;12:90-100

28. Halimi $S$, Vergès B. Adverse effects and safety of SGLT-2 inhibitors Diabetes Metab. 2014:40 (6 Suppl. 1):S28-34.

29. Yang XP, Lai $D$, Zhong XY, et al. Efficacy and safety of canagliflozin in subjects with type 2 diabetes: systematic review and meta-analysis. Eur J Clin Pharmacol. 2014;70:1149-58.

30. Kalra S, Jani A, Ved J, Unnikrishnan AJ. Sodium-glucose cotransporter 2 inhibition and health benefits: the Robin Hood Effect. Indian J Endocr Metab. 2016;20:725-9.

31. Ferrannini E, Muscelli E, Frascerra S, et al. Metabolic response to sodium-glucose cotransporter 2 inhibition in type 2 diabetic patients. J Clin Invest. 2014;124:499-508.

32. Ferrannini G, Hach T, Crowe S, et al. Energy balance after sodium-glucose cotransporter 2 inhibition Diabetes Care. 2015;38:1730-5

33. Ferrannini E, Mark M, Mayoux E. CV protection in the EMPA-REG OUTCOME trial: a "thrifty substrate" hypothesis. Diabetes Care. 2016:39:1108-14.

34. Solini A, Giannini L, Seghieri M, et al. Dapagliflozin acutely improves endothelial dysfunction, reduces aortic stiffness and renal resistive index in type 2 diabetic patients: a pilot study. Cardiovasc Diabetol. 2017;16:138.
35. Sattar N, McLaren J, Kristensen SL, et al. SGLT2 Inhibition and cardiovascular events: why did EMPA-REG Outcomes surprise and what were the likely mechanisms? Diabetologia. 2016:59:1333-9.

36. Mora-Fernández C, Domínguez-Pimentel V, de Fuentes MM et al. Diabetic kidney disease: from physiology to therapeutics. J Physiol. 2014;592:3997-4012.

37. Maltese G, Abou-Saleh A, Gnudi L, et al. Preventing diabetic renal disease: the potential reno-protective effects of SGLT2 inhibitors. Br J Diabetes Vasc Dis. 2015;15:114-8

38. Cherney DZ, Perkins BA, Soleymanlou N, et al. Renal hemodynamic effect of sodium-glucose cotransporter 2 inhibition in patients with type 1 diabetes mellitus. Circulation. 2014;129:587-97.

39. Gnudi L, Karalliedde J. Beat it early: putative renoprotective haemodynamic effects of oral hypoglycemic agents. Nephrol Dial Transplant. 2016;31:1036-43.

40. Wanner C, Inzucchi SE, Lachin JM, et al. Empagliflozin and progression of kidney disease in type 2 diabetes. N Engl J Med.

41. Mudaliar S, Alloju S, Henry RR. Can a shift in fuel energetics explain the beneficial cardiorenal outcomes in the EMPA-REG OUTCOME study? A unifying hypothesis. Diabetes Care. 2016:39:1115-22

42. Thomas MC. Renal effects of dapagliflozin in patients with type 2 diabetes. Ther Adv Endocrinol Metab. 2014;5:53-61.

43. Sano $M$, Takei $M$, Shiraishi $Y$, et al. Increased haematocrit during sodium-glucose cotransporter 2 inhibitor therapy indicates recovery of tubulointerstitial function in diabetic kidneys. J Clin Med Res. 2016;8:844-7.

44. Kimura Y, Kuno A, Tanno M, et al. Canagliflozin, a sodium-glucose cotransporter 2 inhibitor, normalizes renal susceptibility to type 1 cardiorenal syndrome through reduction of renal oxidative stress in diabetic rats. J Diabetes Investig. 2019;10:933-46.

45. Neal B, Perkovic V, Mahaffey KW, et al. Canagliflozin and cardiovascular and renal events in type 2 diabetes. N Engl J Med. 2017;377:644-57.

46. Kosiborod M, Cavender MA, Fu AZ, et al. Lower risk of heart failure and death in patients initiated on sodium-glucose cotransporter- 2 inhibitors versus other glucose lowering drugs: the CVD-REAL study (comparative effectiveness of cardiovascular outcomes in new users of sodium-glucose cotransporter-2 inhibitors). Circulation. 2017;136:249-59.

47. Fioretto P, Zambon A, Rossato M, et al. SGLT2 inhibitors and the diabetic kidney. Diabetes Care. 2016;39(Suppl. 2):S165-71.

48. Zinman B, Wanner C, Lachin JM, et al. Empagliflozin, cardiovascular outcomes, and mortality in type 2 diabetes. N Eng/ J Med. 2015;373:2117-28

49. Zelniker TA, Wiviott SD, Raz I, et al. SGLT2i for primary and secondary prevention of cardiovascular and renal outcomes in type 2 diabetes: a systematic review and meta-analysis of

cardiovascular outcome trias Lancet 2019,39

50. Verma S, Mazer CD, Yan AT, et al. Effect of empagliflozin on left ventricular mass in patients with type 2 diabetes and coronary
artery disease: the EMPA-HEART CardioLink-6 randomized clinical trial. Circulation. 2019;140;1693-702.

51. Perkovic V, Jardine MJ, Neal B, et al. Canaglifozin and renal outcomes in type 2 diabetes and nephropathy. N Eng/ J Med. 2019;380:2295-306

52. Wiviott SD, Raz I, Bonaca MP, et al. Dapagliflozin and cardiovascular outcomes in type 2 diabetes. N Eng/ I Med. 2018;380:347-57.

53. Norhammer A, Bodegard J, Nystrom T, et al. Dapagliflozin and cardiovascular mortality and disease outcomes in a population with type 2 diabetes similar to that of the DECLARE-TIMI 58 trial: a nationwide observational study. Diabetes Obes Metab. trial: a nationwide

54. Buse JB, Wexler DI. Tsapas A, et al. 2019 update to: Management of hyperglycaemia in type 2 diabetes, 2018. A consensus report by the American Diabetes Association (ADA) and the European Association for the Study of Diabetes (EASD). Diabetologia. 2020;63:221-28

55. Arnett DK, Blumenthal RS, Albert MA, et al. 2019 ACC/AHA guideline on the primary prevention of cardiovascular disease. A report of the American College of Cardiology/American Heart Association Task Force on Clinical Practice Guidelines. Circulation. 2019;140:e596-646.

56. Rangaswami J, Bhalla V, Blair JEA, et al. Cardiorenal syndrome: classification, pathophysiology, diagnosis, and treatment strategies. A scientific statement from the American Heart Association. Circulation. 2019:139:e840-78.

57. Savira F, Magaye R, Liew D, et al. Cardiorenal syndrome: multi-organ dysfunction involving the heart, kidney and vasculature. Br J Pharmacol. 2020;177:2906-22.

58. Forman DE, Butler J, Wang Y, et al. Incidence, predictors at admission, and impact of worsening renal function among patients hospitalized with heart failure. J Am Coll Cardiol. 2004;43:61-7.

59. Gottlieb SS, Abraham W, Butler J, et al. The prognostic importance of different definitions of worsening renal function in congestive heart failure. J Card Fail. 2002;8:136-41.

60. Heywood JT, Fonarow GC, Costanzo MR, et al. High prevalence of renal dysfunction and its impact on outcome in 118,465 patients hospitalized with acute decompensated heart failure: a report from the ADHERE database. I Card Fail. 2007;13:422-30.

61. Johnson DW, Craven AM, Isbel NM. Modification of cardiovascular risk in hemodialysis patients: an evidence-based review. Hemodial Int. 2007:11:1-14.

62. Rodriguez E, Arias-Cabrales C, Pascual J. Diabetes mellitus: a single cardiorenal syndrome umbrella. Clin Kidney $\mathrm{J}$.

2020;13:14-6

63. Wanner C. EMPA-REG outcome: the nephrologist's point of view. Am J Med. 2017;130:S63-72.

64. ClinicalTrials.gov. EMPA-KIDNEY (the study of heart and kidney protection with empagliflozin). 2020. Available at: https://clinicaltrials.gov/ct2/show/NCT03594110 (accessed 25 July 2020)

65. ClinicalTrials.gov. A study to evaluate the effect of dapagliflozin on renal outcomes and cardiovascular mortality in patients with chronic kidney disease (Dapa-CKD). 2020. Available at: https://clinicaltrials.gov/ct2/show/NCT03036150 (accessed 25 July 2020).

66. ClinicalTrials.gov. Efficacy and safety of canagliflozin (TA-7284) in patients with diabetic nephropathy. 2020 (accessed 25 July 2020).

67. US Food and Drug Administration. FDA drug safety communication: FDA strengthens kidney warnings for diabetes medicines canagliflozin (Invokana, Invokamet) and dapagliflozin (Farxiga, Xigduo XR). 2016. Available at: www.fda.gov/drugs/drug-safety-and-availability/ at: www.fda.gov/drugs/drug-safety-and-availability/
fda-drug-safety-communication-fda-strengthens-kidneywa-drug-safety-communication-fda-strengthens-kidney25 July 2020)

68. Nadkarni GN, Ferrandino R, Chang A, et al. Acute kidney injury in patients on SGLT2 inhibitors: a propensity-matched analysis. Diabetes Care. 2017;40:1479-85.

69. Donnan JR, Grandy CA, Chibrikov E, et al. Comparative safety of the sodium-glucose co-transporter 2 (SGLT2) inhibitors: a systematic review and meta-analysis. BMJ Open. 2019;9:e022577

70. Chertow GM, Burdick E, Honour M, et al. Acute kidney injury, mortality, length of stay, and costs in hospitalized patients. I Am SOC Nephrol. 2005:16:3365-70.

71. O'Neill J, Fasching A, Pihl L, et al. Acute SGLT inhibition normalizes $\mathrm{O} 2$ tension in the renal cortex but causes hypoxia in the renal medulla in anesthetized control and diabetic rats Am 」 Physiol Renal Physiol. 2015;309:F227-34.

72. Chu C, Lu YP, Yin L, et al. The SGLT2 ihibitor empagliflozin might be a new approach for prevention of acute kidney injury. Kidney Blood Press Res. 2019;44:149-57.

73. Kalra S. Diabetes in special situations: SGLT2 inhibitors in ACS. 2017. Available at: https://diabetes.medicinematters. $\mathrm{com} /$ cardiovascular-disorders/sglt2-inhibitors/diabetes-inspecial-situations-sodium-glucose-co-transporter-2/15199584 (accessed 25 July 2020).

74. Kambara T, Shibata R, Inoue Y, et al. Additional use of SGLT2i in the treatment of acute decompensated heart failure may prevent acute kidney injury. Eur Heart J. 2018;39(Suppl. 1):P272

75. John M, Unnikrishnan AG, Kalra S, et al. Cardiovascular outcome trials for anti-diabetes medication: a holy grail of drug development? Indian Heart I. 2016;68:564-71

76. Kalra S. Cardiorenal syndromes and SGLT2 inhibitor usage. 2019. Available at: https://diabetes.medicinematters.com/ sglt2-inhibitors/cardiovascular-disorders/cardiorenalsyndrome-sglt2-sanjay-kalra/16737656 (accessed 25 July 2020).

77. Janssen Pharmaceuticals, Inc. INVOKANA ${ }^{\oplus}$ (canagliflozin) prescribing information. 2013. Available at: www.janssenlabels. com/package-insert/product-monograph/prescribinginformation/INVOKANA-pi.pdf (accessed 11 September 2020).

78. Boehringer Ingelheim International GmbH. JARDIANCE® (empagliflozin) prescribing information. 2013. Available at: https://docs.boehringer-ingelheim.com/Prescribing\%20 Information/PIs/Jardiance/jardiance.pdf (accessed 11 september 2020).

79. AstraZeneca Pharmaceuticals. FARXIGA ${ }^{\oplus}$ (dapagliflozin) prescribing information. 2014. Available at: www.azpicentral. $\mathrm{com} /$ farxiga/farxiga.pdf (accessed 11 September 2020).

80. Merck Sharp \& Dohme Corp. STEGLATRO TM (ertugliflozin) prescribing information. 2017. Available at: www.merck.com/ product/usa/pi_circulars/s/steglatro/steglatro_pi.pdf (accessed 11 September 2020)

81. O'Connor-Semmes R, Walker $S$, Kapur A, et al. Pharmacokinetics and pharmacodynamics of the SGLT inhibitor remogliflozin eetabonate in subjects with mild and moderate renal impairment. Drug Metab Dispos. 2015;43:1077-83.

82. Vardeny $\mathrm{O}$, Vaduganathan $\mathrm{M}$. Practical guide to prescribing sodium-glucose cotransporter 2 inhibitors for cardiologists. JACC Heart Fail. 2019;7:169-72.

83. Lim VG, Bell RM, Arjun S, et al. SGLT2 inhibitor, canagliflozin attenuates myocardial infarction in the diabetic and nondiabetic heart. JACC Basic Trans/ SC 2019;4:15-26.

84. Zheng S, Roddick A. 66 Acute coronary syndrome in type 2 diabetes treated using SGLT-2 inhibitors, GLP-1 agonists and DPP-4 inhibitors: a comparative network meta-analysis of 127,731 individuals. Heart. 2018;104:A59-60

85. Watanabe K, Miyabe R, Fujjsawa T, et al. Utility of SGLT2 inhibitors in patients with chronic heart failure with diabetes 